\title{
Is There A Compensatory Inverse Association Between Standing Anterior Pelvic Tilt and Craniovertebral Angles when Heavy Backpack Loads are Imposed Onto The Vertebral Colum?
}

Paul $\mathrm{Y}^{1}$, Kurten $\mathrm{M}^{1 *}$, Barnard $\mathrm{M}^{1}$, Ellapen $\mathrm{TJ}^{1}$, Doherty $\mathrm{BD}^{1}$

${ }^{1}$ Tshwane University of Technology, Staatsartillerie Rd, Pretoria West, Pretoria, 0183, South Africa

DOI: $10.36348 /$ jaep.2021.v05i03.005

| Received: 25.01.2021 | Accepted: 03.03.2021 | Published: 30.03 .2021

*Corresponding author: Kurten $\mathrm{M}$

\section{Abstract}

Scholars carrying heavy backpacks incur misaligned vertebral posture and neuro-musculoskeletal discomfort and pain. This report intends to determine whether there is a compensatory inverse relationship between standing anterior pelvic tilt (SAPT) and craniovertebral angles (CVA) when heavy backpacks are carried. A male pubescent carried a backpack weighing $6.2 \mathrm{~kg}$ (17.3\% of his body mass) over both shoulders resting against his vertebral column. The pubescent CVA, SAPT, standing vertex height $(\mathrm{SVH})$ was measure during unloaded, and three loaded phases. Each loaded phase approximately increased from $10 \%, 20 \%$ and $30 \%$ of the pubescent's relative body mass. The incremental backpack masses reduced the SVH and increased the SAPT, while simultaneously reducing CVA. There was a positive relationship between increased backpack mass and SAPT $(\mathrm{r}=+0.9)$. Craniovertebral angle inversely attenuated in response to the heavy backpack loads ( $\mathrm{r}=-0.9)$. These findings indicate that there is an inverse compensatory relationship between SAPT and CVA when carrying heavy backpacks.

Keywords: Anterior pelvic tilt, backpack, craniovertebral angle, scholar.

Copyright () 2021 The Author(s): This is an open-access article distributed under the terms of the Creative Commons Attribution 4.0 International License (CC BY-NC 4.0) which permits unrestricted use, distribution, and reproduction in any medium for non-commercial use provided the original author and source are credited.

\section{INTRODUCTION}

The habitual carrying of backpacks by scholars of all ages has emerged as a societal occupational concern due to the compensatory musculoskeletal ill effects [1, 2]. Voll and Klimt's [3] rule of thumb prescribed scholars to carry a maximum of $10 \%$ of their respective body mass in an attempt to prevent these musculoskeletal ill effects. Steele et al., [4] and Sahib [5] have supported this recommendation, but it has been repudiated by Brackley and Stevenson [6] who recommend a load of $15 \%$. The musculoskeletal occupational concerns include misaligned vertebral posture, neuro-musculoskeletal discomfort and pain resulting in the absence from scholastic and physical activities [1-6]. The misaligned vertebral posture relates to excessive cervical and lumbar lordosis and thoracic kyphosis [7, 8]. Heavy backpack carriage does produce serial distortion of the vertebral column referred to cervico-kypholordotic posture [9]. Cervicokypholordotic posture is the compensatory misaligned vertebral column, having an enlarged cervical lordosis, thoracic kyphosis and lumbar lordosis. Walikca-Cupyrs et al., [10] postulated that heavy backpack carriage initiates an anterior pelvic tilt, which is an attempt of the musculoskeletal system to accommodate the heavy external load carried. The compensatory pelvic rotation impacts on the anterior-posterior weight supporting structure of the vertebral column that has been associated with neuro-musculoskeletal discomfort and pain. There has been numerous studies documenting the change in SAPT $[10,11]$ and CVA $[12,13]$, but, there is a paucity of literature identifying the compensatory relationships among the increasing external backpack loads with changes to SAPT and CVA. This case study intent to document whether a compensatory relationship exists between the magnitude of SAPT and CVA deviations due to carrying various external backpack loads of different masses.

\section{METHODS \\ Participants}

The parent of a male scholar (12 years old) reported that the pubescent displayed a misaligned posture when carrying his school backpack bag [9]. This case study reviewed the six-month progress report post the initial biokinetic consultation and rehabilitative exercise prescription. The scholar indicated that heavier the external mass of the backpack, the greater the lower back and shoulder discomfort he experienced. The 
parent also indicated that heavier the backpack the greater the sagittal plane postural deviation. The pubescent's parent brought the scholar in for a sixmonth post rehabilitation posture analyses consultation. Biokinetics is an exercise therapy vocation that prescribes physical activity and exercises to manage various pathologies [14]. Both pubescent and parent provided child ascent and parental informed consent.

\section{Procedure}

The pubescent's kinathropometrical measures (body mass and SVH), school backpack and biomechanical angles (CVA and SAPT) were measured in the unloaded phase (not carrying backpack) and then during the loaded phases (carrying the backpack). The loaded phase consisted of three trials of different external backpack loads, approximating $10 \%$ incremental loads.

The kinathropometrical measures were recorded as per International Standards for Kinanthropometric Assessments [15]. The biokineticist used Hande et al., [16] protocol to record the pubescent's CVA, and the Starkey's [17] protocol to quantify SAPT. The reduced CVA suggested altered cervical vertebral alignment on thoracic vertebrae representing excessive cervical lordosis. The enlarged SAPT indicated an anterior pelvic rotation (short-arc pelvic-on-femur flexion).

\section{Data Analyses}

Included descriptive (mean and standard deviation) and inferential statistical tests (Pearson correlation) to measure the associations among the incremental external mass of backpack load on SVH, CVA and SAPT. The dynamic CVA and SAPT associations were reviewed to determine compensatory serial distortion of the vertebral column due to school backpack carriage.

\section{RESULTS}

Table-1 exhibited the changes in kinanthropometric and biomechanical angles among the unloaded versus the three loaded phases. The school backpack weighed $6.2 \mathrm{~kg}$ that equates to $17.3 \%$ of the scholar's body mass. The scholar carried the straps of the backpack on both shoulders. A Pearson correlation test was conducted to determine the inverse association between increased SAPT and diminished SVH ( $\mathrm{r}=-0.8)$. As the total body mass increased the SVH and the CVA decreased, but SPA increased. There was a positive association between decreased $\mathrm{SVH}$ and reduction in CVA $(r=+0.9)$ as the total body mass increased. Similarly, there was an inverse relationship between CVA and SAPT $(r=-0.9)$ as external backpack mass increased.

Table-1: Postural changes during unloaded versus loaded phases

\begin{tabular}{|l|l|l|l|l|l|}
\hline Variables & Unloaded & Loaded phase 1 & Loaded phase 2 & Loaded phase 3 & Mean $( \pm$ SD) \\
\hline SVH $(\mathrm{m})$ & 1.435 & 1.425 & 1.42 & 1.405 & $142.1( \pm 1.2)$ \\
\hline Body mass $(\mathrm{kg})$ & 35.8 & 39.6 & 42.9 & 46.7 & $41.25( \pm 4.6)$ \\
\hline *Total body mass (\%) & 100 & 110.6 & 119.8 & 130.4 & $120.2( \pm 60.6)$ \\
\hline CVA $\left(^{\circ}\right)$ & 48 & 40 & 30 & 24 & $35.5( \pm 10.6)$ \\
\hline SAPT $\left(^{\circ}\right)$ & 9 & 28 & 30 & $20.5( \pm 10.1)$ \\
\hline
\end{tabular}

Table-2: The associative changes due to increased total body mass on height, CVA and SPA

\begin{tabular}{|l|l|}
\hline Relationships & Pearson correlation value \\
\hline Relationship between increased total body mass and SVH & -0.9 \\
\hline Relationship between increased total body mass and CVA & -0.9 \\
\hline Relationship between increased total body mass and SAPT & +0.9 \\
\hline Relationship between SVH and CVA & +0.9 \\
\hline Relationship between SVH and SAPT & -0.8 \\
\hline Relationship between CVA and SAPT & -0.9 \\
\hline
\end{tabular}

\section{DISCUSSION}

The findings of this report concur with previous literature that heavy school backpack exceeding $10 \%$ of the incumbent's body mass does produce misaligned posture evident by the increased SAPT and diminished CVA $[7,8,12,13]$. The clinical evidence does support the Ellapen et al., [18] premise that heavy backpack loads does diminish the incumbent's SVH. The diminished SVH is due to the reduced CVA and increased SAPT. The evidence does indicate strong relationship between SAPT and CVA due to the load imposed concurring with Sahib [5]. The pathomechanical cascade of events to achieve this end occurs as follows; increased backpack load anteriorly rotates the pelvis, which produces serial distortion up the closed kinetic chain of the vertebral column resulting a diminished CVA (Figure-1). The inverse reciprocal relationship between increased SAPT and reduced CVA relates to its natural anterior-posterior weight bearing engineering curvature. If the pelvis singularly anteriorly tilted then the pubescent will fall forward as postulated by Ellapen et al., [18]. In order to 
Paul Y et al., J Adv Educ Philos, Mar, 2021; 5(3): 86-89

prevent a forward fall the thoracic vertebrae hyperflexes and the cervical vertebrae drawn forward thereby diminishing the CVA. In order to maintain an erect posture the craniohorizontal angle is increased. This report indicated that at every $10 \%$ incremental backpack load there is an approximately $7 \%$ increased anterior pelvic tilt that produces a compensatory $8 \%$ reduction in CVA. The aforementioned biomechanical findings needs to be empirical validated.

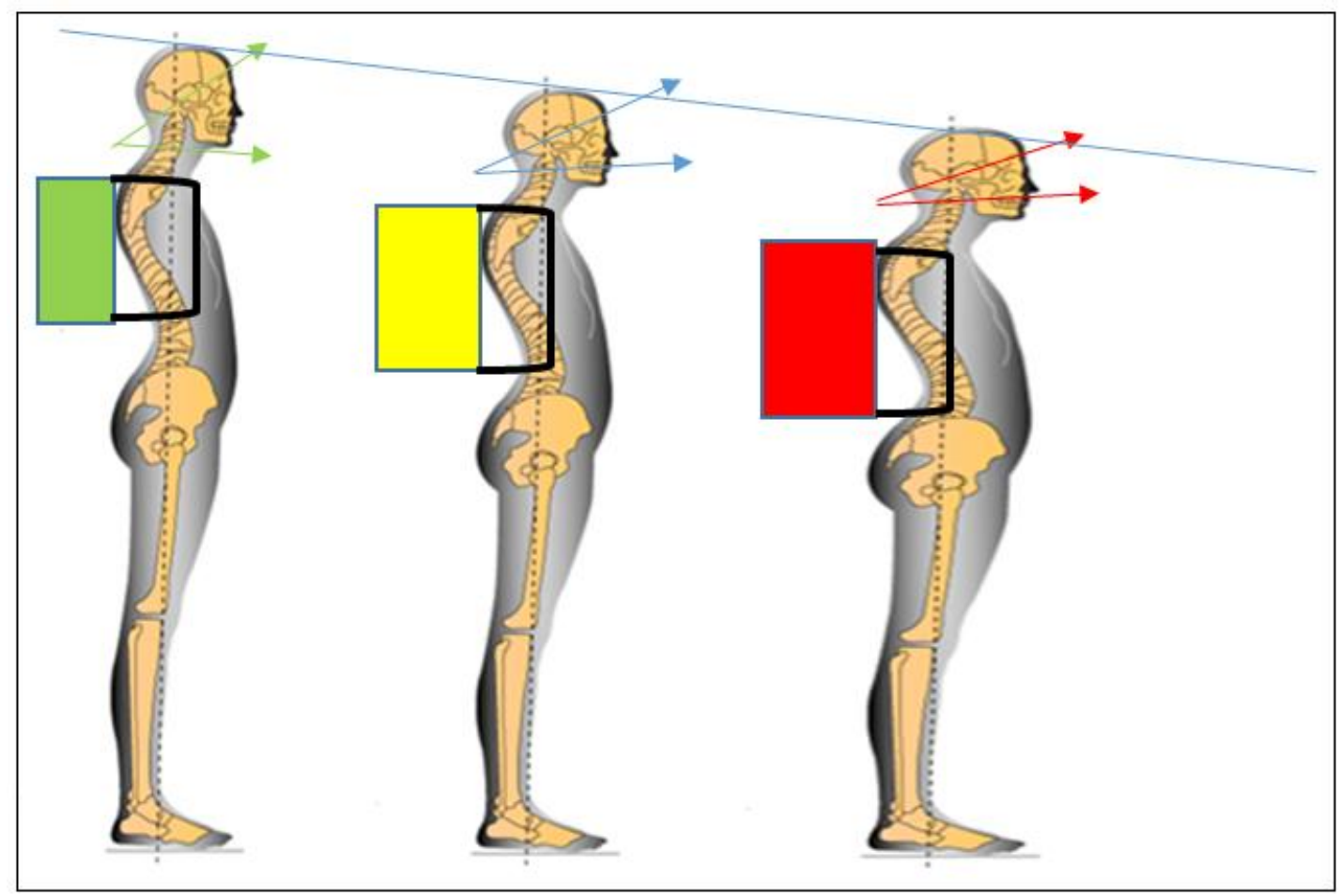

Fig-1: Graphic illustration of the association between increase backpack load and decreases SVH (increasing SAPT and simultaneously reducing CVA) (Paul et al., 2021)

\section{CONCLUSION}

There is a strong inverse relationship between SAPT and CVA when heavy backpacks are imposed onto the vertebral column.

\section{REFERENCE}

1. Dockrell S., Simms C., \& Blake C. (2015). Schoolbag carriage and schoolbag-related musculoskeletal discomfort among primary school children. Applied Ergonomics, 51, 281-90.

2. Azababic, S., Spahic, R., Pranjic, N., \& Mulic, M. (2016). Epidemiology of musculoskeletal disorders in primary school children in Bosnia and Herzegovina, Mater Sociomed, 28(3), 164-167.

3. Voll, H., \& Klimt, F. (1977). Strain in children caused by schoolbags. Offentliche Gesundheitswesen, 39, 369-378.

4. Steele, E., Bialocerkowski, A., \& Grimmer, K. (2003). The postural effects of load carriage on young people: Systematic review. BMC Musculoskeletal Disorders, 4, 12-19.

5. Sahib, M. A. (2016). The effects of schoolbags on the health of students. Karbala Journal of Medicine, 19(1), 2302-2315.

6. Brackley, H., \& Stevenson, J. (2004). Are children's backpack weight limits enough? A critical review of the relevant literature. Spine, 29(19), 2184-2190.

7. Hundekari, J., Chilwant, K., Vedpathak, S., \& Wadee, S. (2013). Does alteration in backpack load affects posture in school children? IORS Journal of dental and Medical Science, 7(4), 7175 .

8. Kistner, F., Fiebert, I., Roach, K., \& Moore, J. (2013). Postural compensation and subjective complaints due to backpack loads and wear time in schoolchildren. Paediatrics Physical Therapy, 1524.

9. Paul, Y., Moraba, L. M., Kurten, M., Barnard, M., \& Ellapen T. J. (2020). Serial distortion patterns of the vertebral column due to schoolbag carriage: A Case Report. Journal of Advances in Education and Philosophy. www.htpp://DOI:10.36348/jaep.2020.v04i10.005

10. Walikca-Cuprys, K., Shalska-Izdebska, R., Rachwal, M., \& Truszcynska, A. (2015). Influence of weight of a school backpack on spinal curvature in the sagittal plane of seven year old children. Bio-Medical Research International, http://dx.doi.org/10.1155/2015/817913

11. Hammill, H. V., Ellapen, T. J., \& Swanepoel, M. (2017). The health impact of schoolbag carriage: A systematic review (2007-2016). African Journal 
for Physical Activity and Health Sciences, 23(2), 245-260.

12. Khallaf, M. E., Fayed, E. E., \& Ashammary, R. A. (2016). 'The effect of schoolbag weight of posture of school children'. Turkish Journal of Physical Medicine and Rehabilitation, 1(62), 16-21. https://doi.org/10.5606/tftrd.2016.12754

13. Goswami, S., Sarkar, L. N., \& Mishra, V. B. (2017). 'The effects of backpack load and carrying duration on head forward inclination of 10-12-year old children'. International Journal of Advanced Educational Research, 2(5), 102-108.

14. Ellapen, T. J., \& Swanepoel, M. (2017). The Evolution of the Profession of Biokinetics. South African Journal of Research in Sport Physical Education Recreation, 39(1): 41-50.

15. Stewart, A., Marfell-Jones, M., Olds, T., \& De Ridder, H. (2011). International standards for anthropometric assessment. Lower Hutt: New Zealand.

16. Hande, D. N., Shinde, N., Khatri, S. M., \& Dangat, P. (2012). The effect of backpack on cervical and shoulder posture in male students of Loni. International Journal of Health Science \& Research, 2(3), 72-79.

17. Starkey, R. (2002). Evaluation of Orthopeadic and Athletic injuries (2nd Ed.), Philadelphia, United States of America: FA Davis Company

18. Ellapen, T. J., Paul, Y., Hammill, H. V., \& Swanepoel, M. (2021). 'Altered cervical posture kinematics imposed by heavy school backpack loading: A literature synopsis (20092019)'.African Journal of Disability, 10(0), a687. https://doi.org/10.4102/ajod. v10i0.687. 